\title{
Caracterização da argila vermiculita expandida: avaliação dos padrões físico-químicos e mineralógicos para aplicação como adsorvente
}

\author{
Characterization of expanded vermiculite clay: \\ an evaluation of the physical-chemical and \\ mineralogical patterns for the application as adsorbent
}

\author{
Luisa Teodoro ${ }^{1}$, Cristiane Regina Budziak Parabocz ${ }^{1}$, \\ Raquel Dalla Costa da Rocha ${ }^{1}$
}

\footnotetext{
${ }^{1}$ Programa de Pós-Graduação em Tecnologia de Processos Químicos e Bioquímicos - PPGTP - DAQUI/UTFPR/PB CEP: 85503-390, Pato Branco, PR, Brasil

e-mail: luisateodoro-@hotmail.com, cristianerb@utfpr.edu.br, raqueldcr@utfpr.edu.br
}

\begin{abstract}
RESUMO
A vermiculita expandida, além de largamente aplicada na construção civil como isolante acústico e retardante de chamas e na agricultura como veículo para fertilizantes, tem sido explorada como possível adsorvente de contaminantes na área ambiental. Dessa forma, objetivou-se caracterizá-la por padrões físico-químicos e mineralógicos. Para tanto foram determinadas as propriedades: ponto de carga zero $\left(\mathrm{pH}_{\mathrm{pcz}}: 8,5\right)$, os sítios ácidos e básicos (carga superficial negativa com presença de grupos ácidos - carboxílicos: 0,9680 $\mathrm{m}_{\mathrm{Eq}} \mathrm{g}^{-1} \mathrm{e}$ fenólicos: 0,0007 $\mathrm{m}_{\mathrm{Eq}} \mathrm{g}^{-1}$ e grupos básicos: $1,5490 \mathrm{~m}_{\mathrm{Eq}} \mathrm{g}^{-1}$ ), capacidade de troca catiônica (CTC: $122 \mathrm{~m}_{\mathrm{Eq}}$ de azul de metileno por100 g de vermiculita expandida), difração de raios X (DRX: alta cristalinidade e pureza), espectrometria de infravermelho com transformada de Fourier (FTIR: característica de filossilicato - deformação axial e angular simétrica de grupamentos O-H, estiramento assimétrico Si-O-Si e Si-O-Al e estiramento Al$\mathrm{O})$, microscopia eletrônica de varredura (MEV: estrutura lamelar típica e vermiforme) e adsorção física de $\mathrm{N}_{2}$ (BET/BJH: sólido mesoporoso - tipo placa, volume de área superficial de $9 \mathrm{~m}^{2} \mathrm{~g}^{-1} \mathrm{~g}$, volume e diâmetro médio do poro de $0,02 \mathrm{~cm}^{3} \mathrm{~g}^{-1}$ e $19 \AA$, respectivamente). Os resultados apontam que a argila vermiculita expandida apresenta-se fortemente como um material adsorvente, possuindo maior afinidade com cátions e moléculas catiônicas e com capacidade de adsorção para moléculas de tamanhos variados.
\end{abstract}

Palavras-chave: Adsorção. Argilomineral. Filossilicato. Lamelares. Mesoporoso.

\begin{abstract}
Expanded vermiculite, in addition to being widely used in civil construction as acoustic insulation and flame retardant and in agriculture as carrier vehicles of fertilizers, has been explored as a possible adsorbent of contaminants in the environmental area. Thus, the objective was to characterize it by physical-chemical and mineralogical standards. For this, the following properties were determined: zero charge point $\left(\mathrm{pH}_{\mathrm{zcp}}: 8.5\right)$, acid and basic sites (negative surface area with the presence of acid groups - carboxylic: $0.9680 \mathrm{~m}_{\mathrm{Eq}} \mathrm{g}^{-1}$ and phenolics $0.0007 \mathrm{~m}_{\mathrm{Eq}} \mathrm{g}^{-1}$ and basic groups: $1.5490 \mathrm{~m}_{\mathrm{Eq}} \mathrm{g}^{-1}$ ), cation exchange capacity (CEC: $122 \mathrm{~m} \mathrm{Eq}$ of methylene blue per $100 \mathrm{~g}$ of expanded vermiculite), X-ray diffraction (XRD: high crystallinity and purity), Fourier transformation infrared spectrometry (FTIR: characteristic of filossilicate, axial and symmetrical angular deformation of O-H groups, asymmetric stretching Si-O-Si and Si-O-Al and stretching Al-O), scanning electronic microscopy (SEM: typical lamellar and vermiform structure) and adsorption of $\mathrm{N}_{2}$ (BET/BJH: mesoporous solid - plate type, volume of surface area of $9 \mathrm{~m}^{2} \mathrm{~g}^{-1}$, pore volume and average pore diameter of 0.02 $\mathrm{cm}^{3} \mathrm{~g}^{-1}$ and $19 \AA$, respectively). The results show that the expanded vermiculite clay is strongly adsorbent, possessing higher affinity with cations and cationic molecules and with adsorption capacity for molecules of different sizes.
\end{abstract}

Keywords: Adsorption. Clayey. Filossilicate. Lamellar. Mesoporous. 


\section{INTRODUÇÃO}

A contaminação de efluentes por resíduos industriais tem sido foco de estudos por muitas décadas, e a busca pelo desenvolvimento sustentável e responsável realça a importância destes, visando minimizar e eliminar os impactos socioambientais das atividades econômicas.

Para remoção dos contaminantes, a adsorção é uma alternativa de alta eficiência, de simples concepção e funcionamento, e pode ser de baixíssimo custo por haver uma grande variedade de materiais passíveis de serem utilizados como adsorventes [1], como é o caso de argilominerais.

Os argilominerais, além de possuírem ampla disponibilidade e baixo custo, tem características intrínsecas que permitem sua utilização como adsorventes de diversos solutos. Dentre estas, destacam-se a elevada capacidade de troca catiônica e área superficial, a estabilidade química e mecânica, elevado número de poros e o diminuto tamanho de suas partículas [2].

Dentre os diversos argilominerais, ressalva-se a vermiculita, que é um mineral micáceo, com composição $\left.(\mathrm{Mg}, \mathrm{Ca})_{0,7}(\mathrm{Mg}, \mathrm{Fe}, \mathrm{Al})_{6,0}\left[(\mathrm{Al}, \mathrm{Si})_{8} \mathrm{O}_{20}\right)\right](\mathrm{OH})_{4} .8 \mathrm{H}_{2} \mathrm{O}$ [3]. Pertencente ao grupo dos filossilicatos argilosos, caracterizados por serem de baixa dureza, entre 2,1 a 2,8 na escala Mohs, e densidade, cujos valores variam entre 800 a $1000 \mathrm{~kg} \mathrm{~m}^{-3}[3,4]$.

Trata-se de uma argila expansiva catiônica, com capacidade de troca catiônica bastante elevada devido às substituições por $\mathrm{Mg}^{2+}$ e $\mathrm{Fe}^{2+}$ dos íons $\mathrm{Al}^{3+}$ nos octaedros e dos íons $\mathrm{Si}^{4+}$ por $\mathrm{Al}^{3+}$ nos tetraedros, que, mesmo que normalmente contrabalançadas, exercem influência direta na capacidade de adsorção da argila $[2$, 5].

As propriedades da vermiculita, químicas e mineralógicas, mostram-se muito promissoras para seu uso no controle ambiental, porém, são pouco conhecidas por parte tanto dos consumidores finais como dos especificadores do produto [6], por isso, o objetivo deste trabalho foi analisar as características adsorventes nos padrões físico químicos e mineralógicos da vermiculita expansiva.

\section{MATERIAIS E MÉTODOS}

A argila vermiculita do tipo expandida superfina foi cedida pela AGROFLOC Brasil Minérios, sendo secada a $105^{\circ} \mathrm{C}$ em estufa (SP-100/42) para remoção de água livre.

A amostra de argila foi caracterizada em relação aos padrões físico químicos e mineralógicos, por meio do Ponto de Carga Zero $\left(\mathrm{pH}_{\mathrm{pcz}}\right.$ ) conforme a metodologia adaptada - variação de $\mathrm{pH}$ [7], a qual consiste em colocar o material sólido em contato com uma solução salina em diferentes valores de $\mathrm{pH}$, quantificando o pH final após 24 horas de contato. Dessa forma, $30 \mathrm{mg}$ de vermiculita expandida foram colocadas em 100 $\mathrm{mL}$ de solução de $\mathrm{NaCl}\left(0,1 \mathrm{~mol} \mathrm{~L}^{-1}\right)$ com $\mathrm{pH}$ previamente ajustado nos valores de 2 a 13 , com auxílio de solução de $\mathrm{HCl}\left(0,1 \mathrm{~mol} \mathrm{~L}^{-1}\right)$ e $\mathrm{NaOH}\left(0,1 \mathrm{~mol} \mathrm{~L}^{-1}\right)$. Após $24 \mathrm{~h}$ em incubadora de bancada tipo Shaker (Te420) com agitação de $100 \mathrm{rpm}$ e temperatura ambiente $\left(25 \pm 2{ }^{\circ} \mathrm{C}\right)$, o pH final de cada ensaio conduzido em triplicata foi aferido por medidor de $\mathrm{pH}$ (Tecnopon).

Também foi determinada a capacidade de troca catiônica (CTC) pelo método da adsorção de azul de metileno $[8,9]$. Para isso, soluções de azul de metileno $\left(0,1 \times 10^{-7}\right.$ a $\left.5,0 \times 10^{-7} \mathrm{~mol} \mathrm{~L}^{-1}\right)$ com pH (sem correção) de 7,7 $\pm 0,2$ foram utilizadas para preparações de curvas de varredura (200 a $\left.800 \mathrm{~cm}^{-1}\right)$ em espectrômetro UV-VIS (Genesys 10s). Posteriormente, em triplicata, $25 \mathrm{~mL}$ de solução de azul de metileno $\left(5,0 \times 10^{-7} \mathrm{~mol}\right.$ $\mathrm{L}^{-1} ; \mathrm{pH}$ : ) foram transferidos em frascos Erlenmeyer com 1,0 g de argila vermiculita expandida e agitados $(150 \mathrm{rpm})$ em incubadora de bancada tipo Shaker (Te-420) por $30 \mathrm{~min}$ em temperatura ambiente $\left(25 \pm 2{ }^{\circ} \mathrm{C}\right)$. Filtrou-se a solução com papel filtro (Whatman GR42) e quantificou-se o azul de metileno remanescente em espectrômetro UV-VIS.

A determinação de Sítios Ácidos e Básicos foi baseada na análise de neutralização [10] e a quantificação de cada grupo ácido pela Equação 1 , sendo que, $\mathrm{V}_{\mathrm{c}}$ volume da solução padrão de $\mathrm{NaOH}\left(0,1 \mathrm{~mol} \mathrm{~L}^{-1}\right)$ gasto na titulação amostra controle $(\mathrm{mL}), \mathrm{V}_{\mathrm{am}}$ volume da solução padrão de $\mathrm{NaOH}\left(0,1 \mathrm{~mol} \mathrm{~L}^{-1}\right)$ gasto na titulação da alíquota da amostra $(\mathrm{mL}), \mathrm{V}_{\mathrm{t}}$ volume total das soluções de $\mathrm{HCl}, \mathrm{NaOH}, \mathrm{NaHCO}_{3} \mathrm{ou} \mathrm{Na}_{2} \mathrm{CO}_{3}$ $(\mathrm{mL}), \mathrm{V}_{\mathrm{a}}$ volume da alíquota do filtrado $(\mathrm{mL})$ e $\mathrm{N}$ concentração da solução de $\mathrm{NaOH}\left(\right.$ eqg $\mathrm{L}^{-1}$ ).

$$
m_{E q}=\frac{V_{t} N\left(V_{a m}-V_{c}\right)}{V_{a}}
$$

Essa metodologia consiste na pesagem de 1,0 g de adsorvente em dois frascos Erlenmeyer, sendo um utilizado para determinar sítios ácidos e outro para sítios básicos. Ao primeiro foram adicionados $50 \mathrm{~mL}$ de $\mathrm{NaOH} 0,1 \mathrm{~mol} \mathrm{~L}^{-1}$, e no segundo $50 \mathrm{~mL}$ de $\mathrm{HCl} 0,1 \mathrm{~mol} \mathrm{~L}^{-1}$ e agitados a $150 \mathrm{rpm}$ em temperatura de $30 \pm$ 
$2{ }^{\circ} \mathrm{C}$ por $48 \mathrm{~h}$ em incubadora de bancada tipo Shaker (Te-420). Após este processo, uma alíquota de $10 \mathrm{~mL}$ de cada uma das soluções foi titulada empregando as correspondentes soluções padrão $0,1 \mathrm{~mol} \mathrm{~L}^{-1} \mathrm{de} \mathrm{HCl}$ e $\mathrm{NaOH}$, a titulação foi conduzida com auxílio de um medidor de pH digital (Tecnopon). Todo o processo de determinação de sítios ácidos e básicos foi realizado com triplicata de amostras.

A determinação dos grupos funcionais da amostra foi realizada por meio de análise de Espectrometria no Infravermelho com Transformada de Fourier (FTIR) realizada em Espectrômetro (Perkin Elmer Frontier), utilizando-se de brometo de potássio $(\mathrm{KBr})$ como agente dispersante. A amostra de vermiculita expandida foi macerada, misturada com $\mathrm{KBr}$ (1:99) e pastilhada. Os espectros foram realizados na região de 4000 a 400 $\mathrm{cm}^{-1}$ com resolução de $2 \mathrm{~cm}^{-1}$ e 32 acumulações.

A análise de Difratometria de Raios X (DRX) foi realizada em difratômetro (Rigaku Mini Flex 600) com intervalo de 3 a $90^{\circ} 2 \theta$ em modo step scan com passo de $0,05^{\circ}$ e duração de 1 passo/segundo.

A Microscopia Eletrônica de Varredura (MEV) foi realizada em microscópio (Hitachi TM3000) nas amplificações de 60, 150 e 500X. E a área superficial foi determinada pelo método de multipontos BET (Brunauer, Emmet e Teller) e BJH (Barret, Joyner e Halenda) que se baseia na adsorção de nitrogênio gasoso a $77 \mathrm{~K}$. A amostra foi inicialmente submetida a uma etapa de tratamento térmico a $300{ }^{\circ} \mathrm{C}$ sob vácuo para remoção da água fisiossorvida por 24 horas, em equipamento Quantachrome NOVA $2000 \mathrm{e}^{-}$.

\section{RESULTADOS E DISCUSSÃO}

A adsorção é um eficaz método de remoção de uma vasta gama de contaminantes de efluentes, principalmente quando a combinação entre adsorvato e adsorvente é a melhor possível. Para tanto, é necessário conhecerse bem a natureza de ambas as partes, fazendo da caracterização prévia uma etapa de suma importância. Segundo MELLO et al. [11] a adsorção depende das características químicas e físicas dos adsorventes, pois tais propriedades estão diretamente relacionadas com os processos de quimissorção e fisissorção do adsorvato.

O ponto de carga zero de um material representa o valor de $\mathrm{pH}$ no qual a adsorção de íons $\mathrm{H}^{+}$e $\mathrm{OH}^{-}$é igual [12]. Controlando o pH do meio pode-se alterar as características de adsorção do adsorvente, tais como a carga de superfície do adsorvente, o grau de ionização da molécula de adsorção e a dissociação dos sítios ativos $[13,14]$.

A vermiculita expandida foi analisada quanto ao $\mathrm{pH}$ no qual a carga superficial do material é nula e esse resultado é observado na Figura 1.

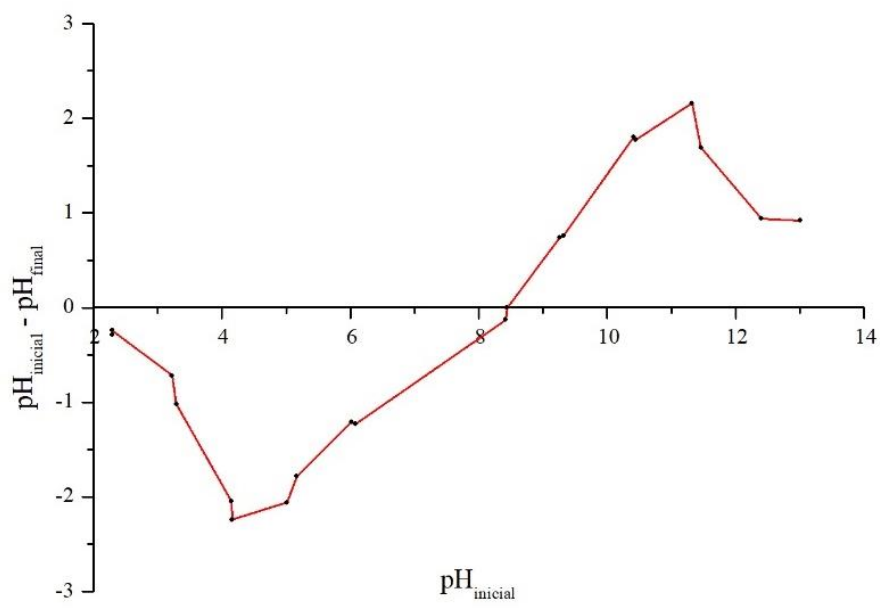

Figura 1: Ponto de carga zero $\left(\mathrm{pH}_{\mathrm{pcz}}\right)$ para a vermiculita expansiva.

O ponto de carga zero aproximado da vermiculita expandida é de $\mathrm{pH} 8,5$, valor próximo do encontrado por NDLOVY et al. [16] $\left(\mathrm{pH}_{\mathrm{pcz}}\right.$ : 8,4) e SILVA et al. [17] $\left(\mathrm{pH}_{\mathrm{pcz}}\right.$ : 8,8). Deste modo, em soluções de pH abaixo do $\mathrm{pH}_{\mathrm{pcz}}$, a vermiculita terá carga superficial positiva e atrairá cargas negativas por atração eletrostática, assim, sendo mais eficaz na remoção de moléculas aniônicas e, em soluções de $\mathrm{pH}$ maior que o $\mathrm{pH}_{\mathrm{pcz}}, \mathrm{a}_{\mathrm{su}}$ perfície, carregada negativamente, adsorverá preferencialmente moléculas catiônicas.

Segundo FAGNANI et al. [18] alterando o pH de uma solução pode-se alterar desde a quantidade adsorvida do adsorvato como também o mecanismo do processo. 
Conforme estudos [17], argilominerais podem ser dissociados formando compostos silanol e aluminol, o que atribui propriedades ácidas e básicas devido a protonação e desprotonação destes dois compostos. Porém quando ocorre o rompimento das ligações $\mathrm{O}-\mathrm{H}$, há um aumento da negatividade da superfície, o que contribui para uma forte interação química entre o argilomineral e o adsorbato.

Os resultados da determinação da massa equivalente $\left(\mathrm{m}_{\mathrm{Eq}} \mathrm{g}^{-1}\right)$ dos grupos ácidos e básicos estão dispostos na Tabela 1.

Tabela 1: Massa equivalente dos grupos superficiais ácidos e básicos determinados na amostra de vermiculita expandida.

\begin{tabular}{c|c|c|c}
\hline \multicolumn{3}{c|}{ GRUPOS ÁCIDOS } & GRUPOS BÁSICOS \\
\hline Carboxílicos $\left(\mathrm{m}_{\mathrm{Eq}} \mathrm{g}^{-1}\right)$ & Lactônicos $\left(\mathrm{m}_{\mathrm{Eq}} \mathrm{g}^{-1}\right)$ & Fenólicos $\left(\mathrm{m}_{\mathrm{Eq}} \mathrm{g}^{-1}\right)$ & $\left(\mathrm{m}_{\mathrm{Eq}} \mathrm{g}^{-1}\right)$ \\
\hline 0,9680 & 0,0000 & 0,0007 & 1,5490 \\
\hline
\end{tabular}

Os sítios básicos podem ser relacionados com a existência de $\mathrm{Si}(\mathrm{OH}) \mathrm{Al}$ [19]. Os grupos ácidos carboxílicos de um sólido tornam-no mais hidrofílico e ácido e diminuem o $\mathrm{pH}_{\mathrm{pcz}}$, enquanto os grupos básicos tornam-no mais hidrofobizado e básico e aumentam o valor do $\mathrm{pH}_{\mathrm{pcz}}$ [20]. Outrossim, a análise evidenciou que existem mais grupos básicos que grupos ácidos, e o $\mathrm{pH}_{\mathrm{pcz}} 8,5$ contribui com esses resultados, demostrando assim uma superfície básica.

Ainda, considerando a teoria de ácidos e bases de Lewis e o pressuposto de que a argila vermiculita possui carga superficial negativa, devido às substituições isomórficas, a existência de grupos básicos, doadores de elétrons, em maior quantidade é corroborada, pois implica em carga superficial negativa.

A Figura 2 apresenta o difratograma da vermiculita expandida.

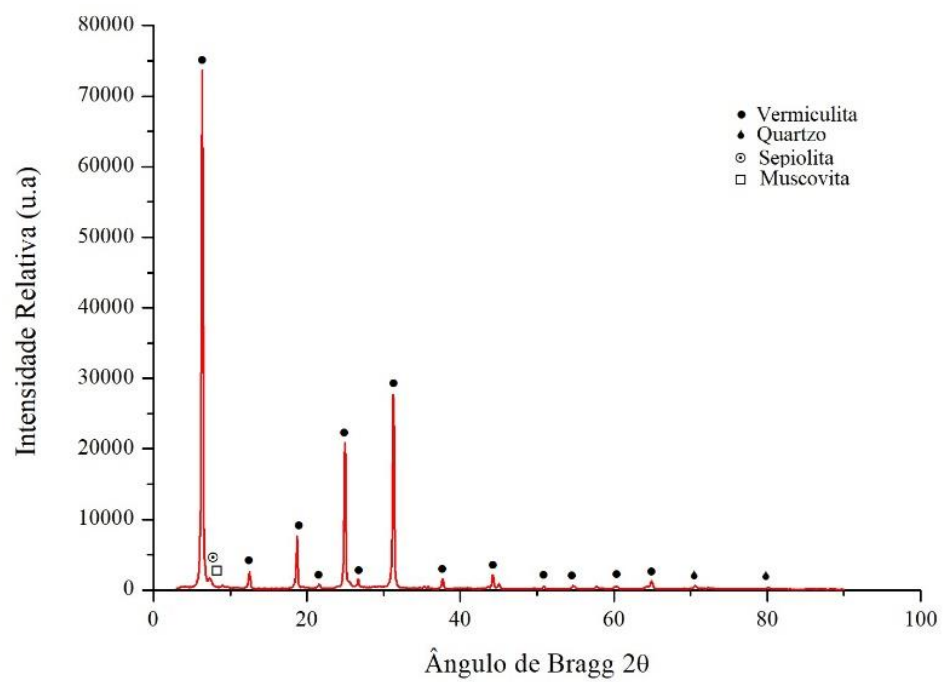

Figura 2: Difratograma de Raios X da vermiculita expandida.

O difratograma obtido demonstra alta pureza e cristalinidade do material, caracterizando que a argila em estudo realmente é vermiculita, o que é confirmado nos estudos encontrados na literatura [21-25]. Observouse na amostra apenas pequenas mudanças de composição, como a presença de sepiolita e muscovita, que podem ser atribuídos ao local de lavra da argila e minerais que a originaram. A cristalinidade de um material adsorvente pode aumentar a adsorção [26, 27].

A Figura 3 corresponde ao espectro de infravermelho com transformada de Fourier obtido nas condições utilizadas, com indicações e interpretações dos comprimentos de ondas dos sinais mais intensos. 


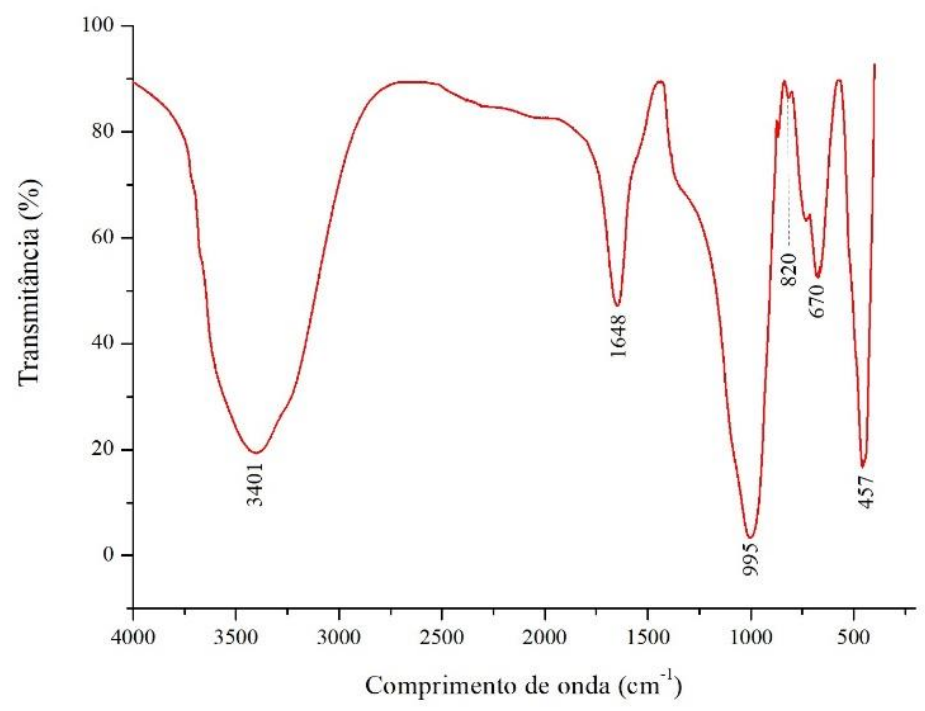

Figura 3: Espectro de FTIR da argila vermiculita expandida dispersa em $\mathrm{KBr}$.

A banda de absorção em $3401 \mathrm{~cm}^{-1}$, larga e intensa em forma de U, é atribuída as vibrações de deformação axial de grupamentos $\mathrm{O}-\mathrm{H}$ dos grupos silanol $(\mathrm{SiOH})$ da estrutura [28]. O sinal em $1648 \mathrm{~cm}^{-1}$ pode ser atribuído a ligação O-H, a sua deformação angular simétrica [29]. A presença deste indica que, apesar do tratamento térmico ao qual a vermiculita foi submetida em seu processo de expansão, há água em sua estrutura, que pode ter sido adsorvida durante o armazenamento sob condições ambientes de temperatura e umidade.

Em $995 \mathrm{~cm}^{-1}$, o sinal de absorção, chamado de "ombro", pode ser atribuído à região de estiramento assimétrico Si-O-Si e Si-O-Al [29, 30]. Em $820 \mathrm{~cm}^{-1}$, o sinal de intensidade leve pode ser atribuído ao estiramento Al-O na folha tetraédrica [23]. A vibração em $670 \mathrm{~cm}^{-1}$ pode ser atribuída a vibrações Al-O e em 465 $\mathrm{cm}^{-1}$ a deformação do grupo $\mathrm{Si}-\mathrm{O}-\mathrm{Si}[23,25]$.

Estes sinais, que são coerentes com a estrutura prevista do filossilicato, também dão indicações sobre a qualidade da adsorção na argila: a existência de grupos $\mathrm{OH}$ livres que permite a ligação adsorvente/adsorvato de caráter químico, bem como os grupos silanol e aluminol [2].

A Figura 4 mostra a amostra de vermiculita expandida nas ampliações de 60, 150 e 500X.
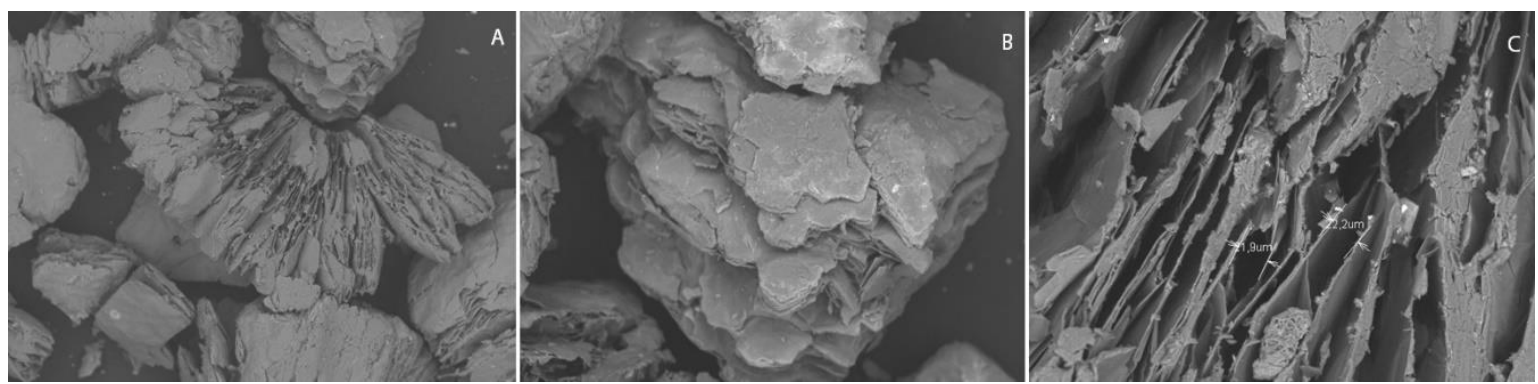

Figura 4: Micrografia da vermiculita expandida com ampliação de A) 60X, B) 150X e C) 500X.

É possível perceber claramente a forma lamelar típica e a estrutura sanfonada vermiforme (Figura 4A). Esta morfologia possibilita que moléculas de variados tamanhos difundam para o interior da vermiculita e adsorvam em sua estrutura [21], observa-se também uma maior área superficial o que possibilita um aumento dos sítios de adsorção.

$\mathrm{Na}$ Figura 4B, observa-se com maior nitidez a face irregular das lamelas. Conforme MORAVIA et al. [31], superfícies irregulares e com rugosidade em argilominerais são atribuídas ao processo de expansão. Os espaços interlamelares com ampliações diferentes (Figura 4C) também são observados na amostra de vermiculita expandida. Esses espaçamentos desiguais podem ser devido a presença irregular de camadas de água adsorvida na amostra [32].

As características texturais da vermiculita expansiva, histerese, área superficial, volume e tamanho de 
poros foram obtidas por meio das isotermas BET e BJH, fazendo-se uma análise importante para o conhecimento do potencial adsortivo, pois tais características estão intimamente relacionadas com a eficiência dos mecanismos de transferência de massa que ocorrem no processo de adsorção.

A Figura 5 mostra a isoterma (Figura 5A) e a distribuição de tamanho dos poros (Figura 5B) obtida para a amostra de vermiculita expandida.
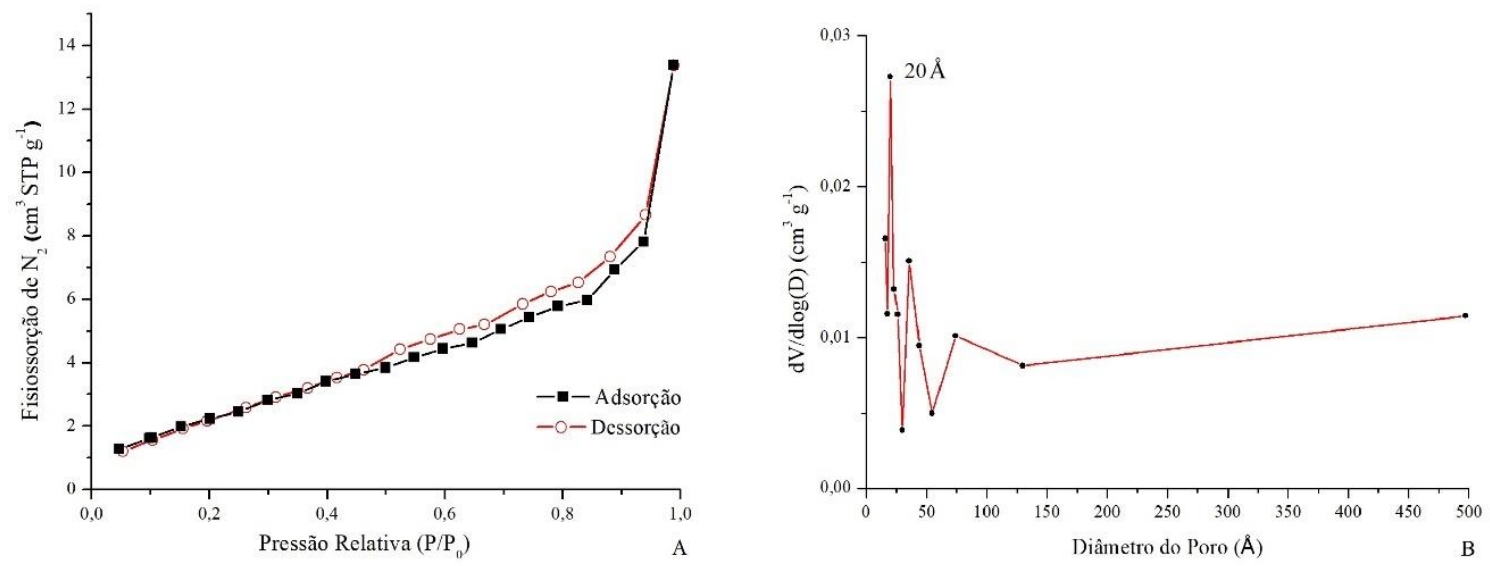

Figura 5: Isotermas (A) de adsorção e dessorção e distribuição de tamanho de poros (B) da vermiculita expandida.

A isoterma apresenta-se próxima ao tipo IV da classificação da IUPAC, caracterizando um sólido mesoporoso, devido aos picos na faixa entre 20 e $500 \AA$ A. Essa isoterma é característica de vários adsorventes e sua parte inicial atribuída à adsorção em mono e multicamada [33]. O que confirma o comportamento geral com a distribuição do tamanho médio de poros (Figura 5B) de 20 Å e a análise BJH em que a média do diâmetro do poro foi de $19 \AA$.

A histerese observada para a vermiculita expandida assemelha-se ao tipo $\mathrm{H} 3$, caracterizando um sólido com aglomerados de partículas formando placas não uniformes em tamanho e forma [34]. Na análise de BET e de BJH foram observados valores de volume de área superficial de $9 \mathrm{~m}^{2} \mathrm{~g}^{-1}$ e volume do poro de $0,02 \mathrm{~cm}^{3}$ $\mathrm{g}^{-1}$.

A capacidade de troca catiônica (CTC) é um dos parâmetros mais importantes quando se trata de argilominerais, pois representa sua capacidade de trocar cátions com fases líquidas [35]. A CTC com azul de metileno permite identificar a capacidade de adsorção dos materiais mesoporosos devido ao tamanho do cátion azul de metileno $\left(\mathrm{C}_{16} \mathrm{H}_{18} \mathrm{~N}_{3} \mathrm{~S}^{+}\right)$que tem tendência a fixar nos mesoporos da superfície do adsorvente [36].

A concentração média de azul de metileno remanescente nos ensaios foi de $0,14 \pm 0,02 \times 10^{-7} \mathrm{~mol} \mathrm{~L}^{-1}$, equivalendo $97,2 \%$ de troca catiônica $\left(122 \mathrm{~m}_{\mathrm{Eq}}\right.$ de azul de metileno por 100 gramas de vermiculita expandida). Segundo TAYLOR [37] na vermiculita é considerado CTC acima de $90 \%$ devido às substituições isomórficas da estrutura. Estudos demonstraram uma variedade de CTC entre 80 a 200 mEq/100 g [38].

\section{CONCLUSÕES}

A vermiculita expandida apresentou propriedades interessantes para a aplicação em adsorção. Observa-se uma forte tendência a interação química devido a existência de grupos $\mathrm{OH}$ livres, aos grupos silanol e aluminol e por ser sólido mesoporoso. Além disso, devido a suas possíveis substituições isomórficas e aos grupos básicos que contribuem para a sua carga superficial negativa, a vermiculita apresenta uma afinidade maior para cátions e moléculas catiônicas.

\section{AGRADECIMENTOS}

Os autores agradecem a AGROFLOC Brasil Minérios pelo material cedido, a Central de Análise UTFPR/PB e o prof. Helton UFPR/Palotina pelos serviços prestados. 


\section{BIBLIOGRAFIA}

[1] DILARRI, G., ALMEIDA, E.J.R., PECORA, H.B., et al.,"Removal of dye toxicity from an aqueous solution using an industrial strain of Saccharomyces cerevisiae", Water, Air, and Soil Pollution, v. 227, n. 8, pp. 227-269, Feb. 2016.

[2] MALANDRINO, M., ABOLlinO, O., GIACOMINO, A., et al., “Adsorption of heavy metals on vermiculite: influence of $\mathrm{pH}$ and organic ligands", Journal of Colloid and Interface Science, v. 299, n. 2, pp. 537-546, Jul. 2006.

[3] MALLA, P.B., Vermiculites-Chemistry, Mineralogy, and Applications, 7 ed., Soil Science Society of America, 2008.

[4] UGARTE, J.F.O., SAMPAIO, J.A., FRANÇA, S.C.A., Vermiculita. Rochas e minerais industriais, 2 ed., Rio de Janeiro, CETEM, 2008.

[5] BHATTACHARYYA, K.G., GUPTA, S.S., "Adsorption of a few heavy metals on natural and modified kaolinite and montmorillonite: a review", Advances in Colloid and Interface Science, v. 140, n. 2, pp. 114131, Jul. 2008

[6] SZNELWAR, J.J., SCALABIN, R., Perfil da vermiculita, Brasília, MME, 2009.

[7] DE FREITAS, F.B.A., DE FREITAS-CÂMARA, M.Y., FREIRE, D.F. "Determinação do PCZ de adsorventes naturais utilizados na remoção de contaminantes em soluções aquosas”, In: Anais do V Encontro Regional de Química \& IV Encontro Nacional de Química [Blucher Chemistry Proceedings], pp. 610-618, São Paulo, Nov. 2015.

[8] HANG, P.T., BRINDLEY, G.W., "Methylene blue absorption by clay minerals. determination of surface areas and cation exchange capacities (clay-organic studies xviii)", Clays and Clay Minerals, v. 18, n. 4, pp. 203-212, Nov. 1970.

[9] BORGES, W.M.S., ARANTES, A.C.C., CASTRO, G.M.M., et al., "Produção, caracterização e avaliação da capacidade adsortiva de carvões ativado em forma de briquete", Matéria, v. 21, n. 4, pp. 930-942, Dez. 2016.

[10] BOEHN, H.P., "Some aspects of the surface chemistry of carbon blacks on other carbons", Carbon, v. 32, n. 5, pp. 759-769, Jan. 1994.

[11] MELO, V.F.B., GAMA, J., FERREIRA, J.M., et al., "Estudo e caracterização de propriedades da argila esmectita na remoção de corantes têxteis", Matéria, v. 18, n. 4, pp. 1467-1482, Dez. 2013.

[12] TEIXEIRA, P.C., DONAGEMMA, G.K., FONTANA, A., et al.,Manual de métodos de

análise de solo, 3 ed., Brasília, EMBRAPA, 2017.

[13] NANDI, B.K., GOSWAMI, A., PURKAIT, M.K., "Adsorption characteristics of brilliant green dye on kaolin”, Journal of Hazardous Materials, v. 161, n. 1, pp. 387-395, Jan. 2009.

[14] RIDA, K., BOURAOUI, S., HADNINE, S., “Adsorption of methylene blue from aqueous solution by kaolin and zeolite”, Applied Clay Science, v. 82, pp. 99-105, Out. 2013.

[15] SILVA, B., TUUGUU, E., COSTA, F., et al., "Permeable biosorbent barrier for wastewater remediation”, Environmental Processes, v. 4, n. 1, pp. 195-206, Nov. 2017.

[16] NDLOVU, B., BECKER, M., FORBES, E., et al., "The influence of phyllosilicate mineralogy on the rheology of mineral slurries", Minerals Engineering, v. 24, n. 12, pp. 1314-1322, Out. 2011.

[17] SILVA-SILVA, M., SANTANA, G.P., "Caulinita: estrutura cristalina, técnicas físicas de estudo e adsorção", Scientia Amazonia, v. 2, n. 3, pp. 54-70, Jan. 2013.

[18] FAGNANI, H.M.C., DEOLIN, M.E., BARROS, M.A.S.D., et al., "Identificação dos mecanismos de sorção em zeólita NaY e sílica gel”, Matéria, v. 22, n. 3, pp. 1-13, Ago. 2017.

[19] CORDEIRO, C.S., SILVA, F.R., WYPICH, F., et al., "Catalisadores heterogêneos para a produção de monoésteres graxos (biodiesel)", Química Nova, v. 34, n. 3, pp. 477-486, Jan. 2013.

[20] MORENO-CASTILHA, C., Eliminación de contaminantes orgânicos de las aguas mediante adsorción em materiales de carbón, Tese de D.Sc., Departamento de Quimica Inorgánica, Universidade de Granada, Madrid, Espana, 2004.

[21] ANTUNES-JUNIOR, A.U., PAZ, A.P., LIMA, E.G., et al., "Avaliação do desempenho de argila vermiculita em reação de transesterificação de óleo de soja e de algodão", In: Anais do I Congresso Nacional de Engenharia de Petróleo, Gás Natural e Biocombustíveis, pp. 1-8, Campina Grande, Mai. 2002. 
[22] LINS, R.R.F., PEIXOTO, R.S.D., MACEDO, D.A., et al., "Efeito da adição de vermiculita expandida nas propriedades tecnológicas de materiais cerâmicos de base argilosa", In: Anais do Congresso Brasileiro de Engenharia e Ciência dos Materiais, pp. 1865 - 1875, Natal, Nov. 2006.

[23] SILVA, A.P.O., PERGHER, S.B.C., PENHA, F.G., et al., "Preparação de argilominerais organofilizados com quitosana”, Perspectiva, v. 37, n. 139, pp. 47-55, Set. 2013.

[24] UGARTE, J.F.O., MONTE, M.B.M., FRANÇA, S.C.A., et al., "Comparação estatística do fator de expansão de concentrados de vermiculita", In: Anais do XX Encontro Nacional de Tratamento de Minérios e Metalurgia Extrativa, pp. 201-208, Florianópolis, Mai. 2005.

[25] JANEK, M., SZÖCS, V., BUGAR, I., "Terahertz time-domain spectroscopy of selected layered silicates", Clays and Clay Minerals, v. 57, n. 4, pp. 416-424, Aug. 2009.

[26] ILHARCO, L.M., SILVA, J.L., GARCIA, A., et al., "Infrared approach to the study of adsorption on cellulose: influence of cellulose crystallinity on the adsorption of benzophenone", Langmuir, v. 13, n. 15, Jul. 1997.

[27] UlibARri, M.A., PAVlOVIC, I., BARRIGA, C., et al., "Adsorption of anionic species on hydrotalcite-like compounds: effect of interlayer anion and crystallinity", Applied Clay Science, v. 18, n. 1, pp. 17-27, Jan. 2001.

[28] SILVERSTEIN, R. M., BASSLER, G. C., MORRILL, T. C., Identificação espectrométrica de compostos orgânicos. 3 ed. Rio de Janeiro, Guanabara Dois S.A., 1979.

[29] DE MORAIS, D.R.P., DELGADO, R.C.O.B., MOTA, A.L.N., et al., "Caracterização de turfa e vermiculita hidrofobizadas por espectroscopia de absorção na região do infravermelho- FTIR", In: Anais do $5^{\circ}$ Encontro Regional de Química \& $4^{\circ}$ Encontro Nacional de Química, pp. 1 - 8, Mossoró, Ago. 2005.

[30] SANTOS, C.P.F., MELO, D.M.A., MELO, M.A.F., et al., "Caracterização e usos de argilas bentonitas e vermiculitas para adsorção de cobre ( II ) em solução", Cerâmica, v. 48, n. 308, pp. 178-182, Out/Nov.Dez. 2002.

[31] MORAVIA, W.G., OLIVEIRA, C.A.S., GUMIERI, A.G., et al., "Caracterização microestrutural da argila expandida para aplicação como agregado em concreto estrutural leve", Cerâmica, v. 52, n. 322, pp. 193-199, Out/Nov.Dez. 2006.

[32] SAMPAIO, E., Mineralogia do solo. 1 ed. Évora, Universidade de Évora, 2006.

[33] IUPAC The PubChem Project, "Manual of symbols and terminology for physiochemical quantities and units - appendix II.", Pure \& Appl Chem, v. 46, pp. 71-90, 1976.

[34] LEOFANTI, G., PADOVAN, M., TOZZOLA, G., et al., "Surface area and pore texture of catalysts", Catalysis Today, v. 41, n. 1, pp. 207-219, May. 1988.

[35] MILOSEVIC, M., LOGAR, M., DOJCINOVIC, B., et al., "Suitability of the methylene blue test for determination of cation exchange capacity of clay minerals related to ammonium acetate method", Geophysical Research Abstracts, v. 17, pp. 2174, Apr. 2015.

[36] JAPANESE INDUSTRIAL STANDARD (JIS) Test methods for activated carbon, Tóquio, Japanese Standards Association, 1992.

[37] TAYLOR, R.K., "Cation exchange in clays and mudrocks by methylene blue", Journal of Chemical Technology and Biotechnology, v. 35, n. 4, pp. 195-207, Feb. 1985.

[38] PERGHER, S.B.C., DETONI, C., MIGNONI, M.L., Materiais laminares pilarizados - preparação, caracterização, propriedades e aplicações, 1 ed., Erechim, Edifapes, 2005.

\section{ORCID}

Luisa Teodoro

Cristiane Regina Budziak Parabocz

Raquel Dalla Costa da Rocha https://orcid.org/0000-0002-0508-8991

https://orcid.org/0000-0002-8905-8553

https://orcid.org/0000-0002-0440-7583 